\title{
The aerodynamic effects of wing rotation and a revised quasi-steady model of flapping flight
}

\author{
Sanjay P. Sane* and Michael H. Dickinson \\ Department of Integrative Biology, University of California, Berkeley, CA 94720, USA \\ *e-mail: sane@socrates.berkeley.edu
}

Accepted 30 January 2002

\begin{abstract}
Summary
We used a dynamically scaled model insect to measure the rotational forces produced by a flapping insect wing. A steadily translating wing was rotated at a range of constant angular velocities, and the resulting aerodynamic forces were measured using a sensor attached to the base of the wing. These instantaneous forces were compared with quasi-steady estimates based on translational force coefficients. Because translational and rotational velocities were constant, the wing inertia was negligible, and any difference between measured forces and estimates based on translational force coefficients could be attributed to the aerodynamic effects of wing rotation. By factoring out the geometry and kinematics of the wings from the rotational forces, we determined rotational force coefficients for a range of angular velocities and different

to the three-dimensional case using blade element theory. As predicted by theory, the rotational coefficient varied linearly with the position of the rotational axis for all angular velocities measured. The coefficient also, however, varied with angular velocity, in contrast to theoretical predictions. Using the measured rotational coefficients, we modified a standard quasi-steady model of insect flight to include rotational forces, translational forces and the added mass inertia. The revised model predicts the time course of force generation for several different patterns of flapping kinematics more accurately than a model based solely on translational force coefficients. By subtracting the improved quasi-steady estimates from the measured forces, we isolated the aerodynamic forces due to wake capture.
\end{abstract} axes of rotation. The measured coefficients were compared with a mathematical model developed for twodimensional motions in inviscid fluids, which we adapted
Key words: quasi-steady, model, insect, flight, aerodynamics, wing rotation, kinematics, flapping, rotational forces.

\section{Introduction}

In most insects, the kinematics of flapping flight consists of two translational phases during which the wings sweep through the air with relatively slow changes in the angle of attack, followed by rapid rotations at the end of each stroke. These wing flips, termed 'pronation' for the upstroke-to-downstroke transition and 'supination' for the downstroke-to-upstroke transition, allow insects to maintain a positive angle of attack and thus to generate lift during both forward and reverse strokes. An understanding of the actual aerodynamic significance of these wing rotations has long been hindered by a lack of precise instantaneous force measurements on flapping airfoils. However, with recent advances in our knowledge of the instantaneous forces on wings (Dickinson et al., 1999), it is possible to characterize the role of wing rotation and to incorporate rotation in existing quasi-steady models of insect flight.

Aerodynamic theorists have long recognized the importance of airfoil rotation in the context of fluttering wings. Munk (1925) predicted that, when two-dimensional airfoils translate while simultaneously rotating with small amplitudes, additional circulation is required to maintain the Kutta condition at the trailing edge. He calculated that the magnitude of rotational circulation should depend on the axis of rotation such that, when the axis of rotation crosses a critical point along the chord, the circulation will reverse sign. Thus, there exists a critical axis on the wing about which rotation contributes no net circulation. The relative position of the rotational axis with respect to this critical axis determines whether rotational circulation enhances or attenuates the lift generated via translation.

These ideas were further developed by Glauert (1929) and Theodorsen (1935) and later by Fung (1969), who proposed a quasi-steady model for flutter and predicted that the critical axis resides at a distance of 0.75 chord lengths from the leading edge. Reid (1927), Silverstein and Joyner (1939) and Halfman (1951) provided experimental support for these models by demonstrating that oscillating airfoils placed in a steady air stream generate aerodynamic forces that differ from the steadystate case in accordance with the theoretical predictions. Most notably, Farren (1935) investigated how forces varied with both increasing and decreasing angle of attack on an airfoil placed in a wind tunnel and showed that, when the angle of 
attack increases, aerodynamic force coefficients are enhanced compared with corresponding steady-state values. In contrast, when the angle of attack decreases, the force coefficients are lower than the steady-state values. Because these experiments aimed to simulate inviscid conditions, they were performed at high Reynolds numbers and may not be directly applicable to the low-to-intermediate Reynolds numbers relevant to insect flight. In addition, these previous experiments explored a range of angular velocities of wing rotation that are at least one order of magnitude lower than those used by insects when pronating and supinating their wings.

To address the role of wing rotation during insect flight, Bennett (1970) conducted experiments with a dynamically scaled flapping model wing and showed that rotations alter aerodynamic forces at Reynolds numbers in the range $10^{2}$ to approximately $10^{3}$. In a detailed overview of insect flight aerodynamics, Ellington (1984c) proposed a scheme to include wing rotation with translation in quasi-steady models. However, in the one instance in which wing rotation was incorporated into a quasi-steady framework and tested against measurements, instantaneous forces were monitored on the body of the tethered insect rather than on individual wings (Wilkin and Williams, 1993). As a result, it was difficult to separate aerodynamic forces from inertial forces and to distinguish among the various sources of lift. Recently, direct measurements of aerodynamic forces on the wings of a dynamically scaled model fruit fly Drosophila melanogaster showed that, during the stroke, the wings produce aerodynamic forces in excess of those predicted by steady-state translation. The increasing angle of incidence prior to stroke reversal augmented instantaneous values of lift, whereas the decreasing angle of incidence after stroke reversal attenuated lift below quasi-steady translational predictions (Dickinson et al., 1999). These results confirmed similar findings by Bennett (1970) based on stroke-averaged values of the lift estimated from the flow velocity measured using a mechanical model of the cockshafer Melolontha vulgari.

With recent advances in computational fluid dynamics (CFD), efforts in mathematical modeling of insect flight have moved away from quasi-steady approximations to full-scale Navier-Stokes simulations of fluid dynamics (Liu et al., 1998; Wang, 2000). However, while CFD models show promise as an important tool, their application to insect flight is as yet limited by the complex nature of three-dimensional flows in intermediate-to-low Reynolds numbers and by the computational resources required to simulate such conditions. Although not as rigorous as computational simulations, quasisteady models continue to offer a tractable means of calculating instantaneous forces from measured kinematics, are readily applicable to the analysis of energy and power requirements and are more easily incorporated into dynamic control models of insect flight. As we begin to identify the various mechanisms by which insect wings generate aerodynamic forces, it is worthwhile revisiting quasi-steady models to re-assess their validity after taking all known aerodynamic phenomena into account.
In this study, we attempt to characterize the effects of wing rotation on aerodynamic force generation under conditions that are appropriate for analysis of insect flight. At constant translational wing velocity, we vary both the angular velocity and the axis of wing rotation of a dynamically scaled model wing and measure the corresponding rotational force coefficients. We compare these values with a theoretical model based on a two-dimensional rotating flat plate. Although subtle differences do exist, the theoretical predictions provide a reasonably close fit to measured values of rotational force coefficients. By incorporating the rotational effects into a translational quasi-steady model of flapping flight, the predictions of instantaneous forces on insect wings are substantially improved. This revised quasi-steady model may help researchers to better estimate the time course of the forces generated by wings flapping with arbitrary kinematics. Further, because the improved quasi-steady model accurately accounts for both translational and rotational components, as well as the added mass inertia, it may be used to selectively isolate unsteady forces such as those due to wing/wake interactions.

\section{Materials and methods}

The design of the mechanical model used in this study and the procedures for data analyses are identical to those described previously (Dickinson et al., 1999; Sane and Dickinson, 2001). We used an isometrically enlarged planform of a Drosophila melanogaster wing made from a $2.3 \mathrm{~mm}$ thick acrylic sheet, with a length of $25 \mathrm{~cm}$ and mean chord length of $6.7 \mathrm{~cm}$ (calculated aspect ratio of the wing pair 7.5) (see Ellington, 1984b). The proximal edge of the wing was equipped with multiple, equally spaced slots, allowing us to change the axis of rotation (Fig. 1A). Through a pair of these multiple slots, the wing was attached to a two-dimensional force transducer that measured forces normal and parallel to the wing surface. The wings, force sensor and gearbox were immersed in a tank of mineral oil with a kinematic viscosity of $120 \mathrm{cSt}$ $\left(1.2 \times 10^{-4} \mathrm{~m}^{2} \mathrm{~s}^{-1}\right)$ at room temperature (approximately $\left.25^{\circ} \mathrm{C}\right)$. All experiments were conducted at a Reynolds number of approximately 115, calculated as described in Ellington (1984d).

In addition to aerodynamic forces, the sensor at the base of the wing measures the forces due to gravity and the inertia of the wing and sensor. To remove the effect of gravity, we measured the weight of the sensor and wing mass and subtracted it from the measured force traces. The contribution of the inertial effects of the wing mass and sensor was examined by replacing the Plexiglas wing with a brass knob of the same mass. We also ensured that the centers of mass of both the Plexiglas wing and the brass model were identical. Because the compact brass knob generates negligible aerodynamic forces compared with the Plexiglas wing, the measured forces for the brass model may be ascribed entirely to gravity and inertia. Compared with the gravitational contribution, the inertia of the wing was very small and was therefore ignored. 
A

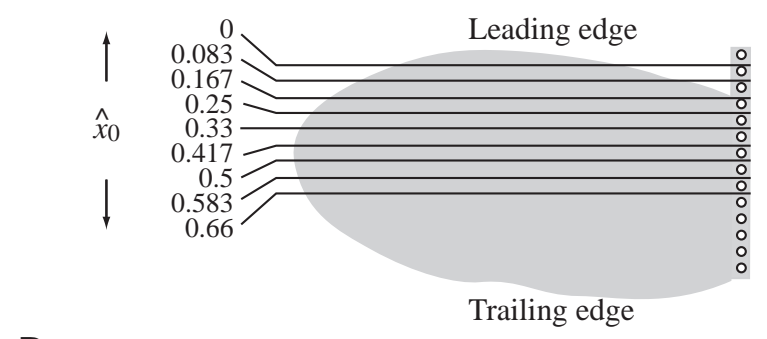

B
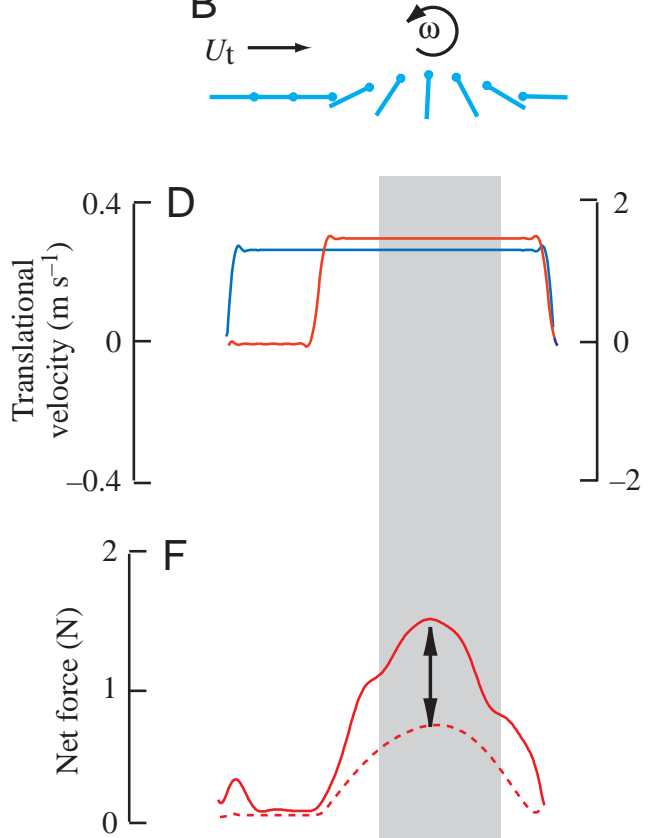

0
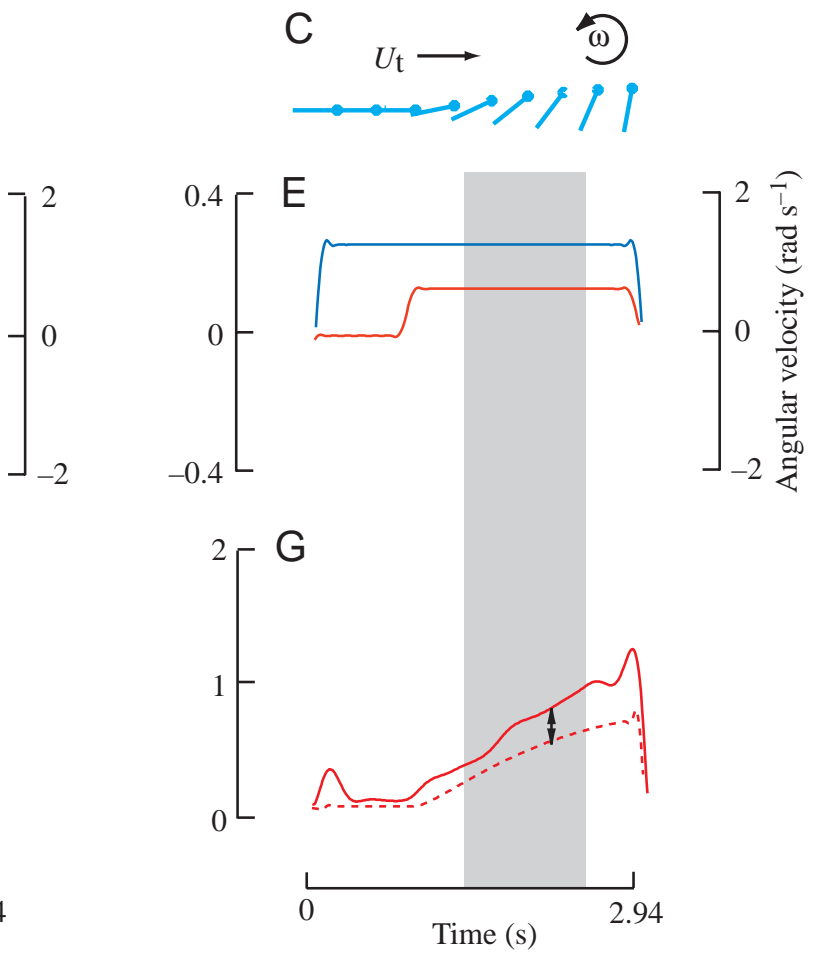

Fig. 1. Wing design and the experimental method. (A) The wing planform used for all experiments. The wing was scaled directly from a Drosophila melanogaster wing and equipped with multiple slots at the base to allow the axis of rotation to be changed. The leading edge corresponds to a non-dimensional rotational axis $\left(\hat{x}_{0}\right)$ value of 0 , whereas the trailing edge corresponds to a value of 1 . (B,C) Two-dimensional cartoons showing the kinematics of rotation and translation. The wing translates from left to right at a velocity $U_{\mathrm{t}}$ and rotates about a fixed axis of rotation with varying angular velocity, $\omega$. The leading edge of the wing is indicated by a filled circle. (D,E) Kinematic variables as a function of time. Translational velocity is shown in blue, rotational velocity in red. Data are shown for two representative rotational velocities of 1.5 rad s ${ }^{-1}$ (D) and $0.667 \mathrm{rad} \mathrm{s}^{-1}$ (E). In both cases, the translational velocity is $0.272 \mathrm{~m} \mathrm{~s}^{-1}$. (F,G) Net aerodynamic forces as a function of time. The continuous red line indicates the measured forces and the dotted red line indicates the quasi-steady translational estimates. The difference between these traces (double-headed arrow) is used to calculate rotational force coefficients over the shaded region. The early peaks in both force traces are due to inertial transients caused by rapid acceleration of the wing at the start of each trial. Similar inertial effects also occur as a result of rapid rotational acceleration, as is evident in the force traces. Although detectable, these effects are small in comparison with the circulatory forces.

The force data were filtered on line using an active four-pole Bessel filter with a frequency cut-off at $10 \mathrm{~Hz}$. They were further processed off line using a low-pass digital Butterworth filter with a zero phase delay and a cut-off at $3 \mathrm{~Hz}$, which was 17.6 times the wing stroke frequency. Increasing the cut-off frequency of the digital filter amplified the influence of jitter resulting from the high-frequency motor steps but did not alter the time course of the recordings.

\section{Stroke kinematics}

We performed 171 separate experiments. In all these experiments, the wing began moving from rest at zero angle of attack and accelerated to a constant translational velocity within $0.05 \mathrm{~s}$. Each stroke was completed in $2.94 \mathrm{~s}$. After attaining a constant translational velocity, the wing rotated with constant angular velocity (Fig. 1B-E). To avoid the influence of wake vorticity from previous strokes on force production (Birch and Dickinson, 2001), only forces measured during the initial forward stroke were used to calculate rotational coefficients (Fig. 1F,G). The absolute angular velocity was systematically varied in separate trials from 0 to $1.5 \mathrm{rad} \mathrm{s}^{-1}$ in increments of $0.085 \mathrm{rad} \mathrm{s}^{-1}$. The absolute 
translational velocity of the wing in these experiments was $0.272 \mathrm{~m} \mathrm{~s}^{-1}$. To compare our results with those in the literature (Walker, 1931; Kramer, 1932; Fung, 1969), we also express angular velocity in non-dimensional terms $(\hat{\omega})$ (after Ellington, 1984c):

$$
\hat{\omega}=\omega \bar{c} / U_{\mathrm{t}}
$$

where $\omega$ is the absolute angular velocity, $\bar{c}$ is the mean chord length and $U_{\mathrm{t}}$ is the wing tip velocity. The range of $\hat{\omega}$ values explored in these experiments was $0-0.374$.

By attaching the wing to the sensor in different positions, we conducted a series of angular velocity trials over a range of non-dimensional axes of rotation $\left(\hat{x}_{0}\right)$ from 0 to 0.66 in increments of 0.083 , where 0 indicates the leading edge and 1 indicates the trailing edge. We could not examine values of $\hat{x}_{0}$ greater than 0.66 because, at these locations, the large moments around the rotational axis threatened to damage the wing sensor at high values of angular velocity.

\section{Components of the quasi-steady model}

In the absence of skin friction, the instantaneous forces generated by a thin, flapping wing may be represented as the sum of four force components, each acting normal to the wing surface:

$$
F_{\text {inst }}=F_{\mathrm{a}}+F_{\text {trans }}+F_{\text {rot }}+F_{\text {wc }},
$$

where $F_{\text {inst }}$ is the instantaneous aerodynamic force on the wing, $F_{\mathrm{a}}$ is the force due to the inertia of the added mass of the fluid, $F_{\text {trans }}$ is the instantaneous translational force, $F_{\text {rot }}$ is the rotational force and $F_{\mathrm{wc}}$ is the force due to wake capture. Note that each term of this quasi-steady model is implicitly, but not explicitly, dependent on time. Thus, any time-dependence of these force components arises only from the time-dependence of the kinematics.

For two-dimensional motion in an inviscid fluid, the first term, $F_{\mathrm{a}}$, is calculated for each blade element and integrated along the span of the wing to estimate the force on a threedimensional airfoil (Sane and Dickinson, 2001) (see Sedov, 1965):

$$
\begin{aligned}
F_{\mathrm{a}}=\rho \frac{\pi}{4} R^{2} \bar{c}^{2}(\ddot{\phi} \sin \alpha+\dot{\phi} \dot{\alpha} \cos \alpha) & \int_{0}^{1} \hat{r} \hat{c}^{2}(\hat{r}) \mathrm{d} \hat{r} \\
& -\ddot{\alpha} \rho \frac{\pi}{16} \bar{c}^{3} R \int_{0}^{1} \hat{c}^{2}(\hat{r}) \mathrm{d} \hat{r},
\end{aligned}
$$

where $\rho$ is the fluid density, $R$ is the wing length, $\hat{r}$ is the nondimensional radial position along the wing, $\hat{c}(\hat{r})$ is the nondimensional chord length (Ellington, 1984b), $\phi$ is the angular position of the wing and $\alpha$ is the morphological angle of attack. Note that, for a wing of infinitesimal thickness, the force due to added mass inertia acts normal to the wing surface.

The quasi-steady translational estimate for the net force was obtained through vector addition of the mutually orthogonal lift and drag estimates:

$$
F_{\text {trans }}=\frac{\rho S U_{\mathrm{t}}^{2} \hat{r}_{2}^{2}(S)}{2}\left[C_{\mathrm{Lt}^{2}}(\alpha)+C_{\mathrm{Dt}^{2}}(\alpha)\right]^{1 / 2},
$$

where $S$ is the projected surface area of the wing and $\hat{r}_{2}^{2}(S)$ is the non-dimensional second moment of wing area (Ellington, 1984b). Note that, in these equations, $\alpha$ and $U_{\mathrm{t}}$ are the only terms that vary throughout the stroke. Values for the lift and drag coefficients, $C_{\mathrm{Lt}}(\alpha)$ and $C_{\mathrm{Dt}}(\alpha)$, have been previously measured for the model wing used in this study and are accurately fitted by the following equations (Dickinson et al., 1999):

$$
C_{\mathrm{Lt}}(\alpha)=0.225+1.58 \sin (2.13 \alpha-7.2)
$$

and

$$
C_{\operatorname{Dt}}(\alpha)=1.92-1.55 \cos (2.04 \alpha-9.82),
$$

where $\alpha$ is in degrees. These equations allow us to determine the forces that arise from the translational mechanism of dynamic stall.

To measure the contribution of rotational forces, we programmed the stroke kinematic patterns as described above. In the absence of added mass inertia or wake capture effects, we can rewrite equation 2 as:

$$
F_{\text {rot }}=F_{\text {inst }}-F_{\text {trans }} .
$$

Thus, we isolate rotational force by subtracting a quasi-steady, translational estimate from the forces measured at a time when inertia and wake capture are insubstantial (Fig. 1F,G). This force difference may be used to derive the experimental values for rotational coefficients. In subsequent sections, experimentally determined values for rotational forces will be denoted by $F_{\text {rot,exp }}$, whereas theoretically determined values for rotational forces will be denoted by $F_{\text {rot,theo }}$

\section{Theoretical estimation of rotational forces}

A quasi-steady treatment of the aerodynamic force due to wing rotation was derived by Fung (1969) (see also Theodorsen, 1935; Sedov, 1965; Ellington, 1984c) for small-amplitude flutter on thin, rigid wings. For a two-dimensional wing of chord length $c$, flapping in an inviscid fluid and rotating with an angular velocity $\omega$ (where $\omega=\dot{\alpha}$ ) around an axis at $\hat{x}_{0}$, the quasi-steady assumption requires two boundary conditions to be satisfied at every instant. First, the direction of fluid velocity near the surface must be equal to the slope of the airfoil surface, which is equivalent to requiring that no fluid travels across the surface of the wing. Second, the vorticity generated by the trailing edge chord element must be zero (Kutta condition). The resultant theoretical value of rotational circulation, $\Gamma_{\text {rot,theo, that satisfies }}$ these boundary conditions is given by:

$$
\Gamma_{\text {rot,theo }}=C_{\text {rot,theo }} \omega c^{2},
$$

where the theoretical value of rotational coefficient, $C_{\text {rot,theo, }}$ is given by:

$$
C_{\text {rot,theo }}=\pi\left(0.75-\hat{x}_{0}\right) .
$$

As $\hat{x}_{0}$ varies from 0 to $1, C_{\text {rot,theo }}$ changes sign at $\hat{x}_{0}=0.75$. Thus, a $\hat{x}_{0}$ value of 0.75 represents the critical axis around which the wing generates no force as it rotates.

In standard Kutta-Jukowski theory, the approximation of a small angle of attack applies, and there is no net drag. Consequently, the lift per unit span on the airfoil equals the 
total aerodynamic force per unit span. Assuming that the theory holds true for large angles of attack, we can use the Kutta-Jukowski equation to relate instantaneous rotational circulation to the rotational component of the total aerodynamic force:

$$
F^{\prime}{ }^{\prime} \text { tot,theo }(t)=\rho U_{\infty} \Gamma_{\text {rot,theo }}(t),
$$

where $F^{\prime}$ rot,theo $(t)$ is the theoretically estimated net force per unit span due to rotation, $6 t$ is time, $\rho$ is the density of the fluid medium, $U_{\infty}$ is the free-stream velocity and $\Gamma_{\text {rot,theo }}(t)$ is the theoretical value for rotational circulation around the wing. Because the net force acts perpendicular to the airfoil surface rather than normal to the wing motion, lift and drag emerge as orthogonal components of the net force.

By combining equations 8 and 10 , and replacing $U_{\infty}$ with $U_{\mathrm{t}}$ as required for a non-dimensional form of blade element analysis, the net estimated rotational force on a flapping, rotating wing of finite span is:

$$
F_{\text {rot,theo }}=C_{\text {rot,theo } \rho} U_{\mathrm{t}} \omega \bar{c}^{2} R \int_{0}^{1} \hat{r} \hat{c}^{2}(\hat{r}) \mathrm{d} \hat{r}
$$

\section{Experimental determination of rotational force coefficients}

To measure the rotational force coefficient, we may substitute for $F_{\text {rot,theo }}$ and $C_{\text {rot,theo }}$ in equation 11 with the corresponding symbols $F_{\text {rot,exp }}$ and $C_{\text {rot,exp }}$ for experimental values:

$$
C_{\text {rot,exp }}=F_{\text {rot,exp }} / \rho U_{\mathrm{t}} \omega \bar{c}^{2} R \int_{0}^{1} \hat{r} \hat{c}^{2}(\hat{r}) \mathrm{d} \hat{r} .
$$

This equation allows us to evaluate coefficients directly from measured rotational forces and compare them with the theoretical estimates given by equation 9 . Note that, although the theoretical estimates in equation 9 depend on all the standard assumptions of Kutta-Jukowski theory, equation 12 is independent of any such assumptions. As a result, the effects of a finite wing span and other aspects of three-dimensional flow are contained in measured values of instantaneous $C_{\text {rotexpp. To }}$ attain a single value of $C_{\text {rotexp }}$ in each trial, we averaged measurements over a temporal window, as shown in Fig. 1D-G.

The sign of the rotational circulation, as well as the rotational forces, is either the same as, or opposite to, that of the circulation due to translation, depending on whether the angle of attack increases or decreases over time. In the experimental situation described above, positive rotation is simply the mirror image about the horizontal axis of the negative rotation case. As a result, separate experiments were not required for measuring coefficients during positive and negative rotation.

\section{Results}

\section{Rotational coefficients versus angular velocity}

Fig. 2A illustrates the dependence of the measured rotational force coefficient $\left(C_{\text {rot,exp }}\right)$ on the non-dimensional angular velocity of wing rotation $(\hat{\omega})$ for a series of nondimensional axes of rotation $\left(\hat{x}_{0}\right)$. For values of $\hat{\omega}$ less than 0.123 ( or $\omega<0.5 \mathrm{rad} \mathrm{s}^{-1}$ ), values of $C_{\text {rot, exp }}$ tend towards infinity (see equation 12) and should be ignored. For values of $\hat{\omega}$ above $0.123, C_{\text {rotexp }}$ rises linearly with $\hat{\omega}$. This trend contradicts the theoretical prediction (equation 9) that $C_{\text {rotexp }}$ should remain constant with respect to $\hat{\omega}$. However, for axes of rotation nearer the trailing edge, the dependence of $C_{\text {rot,exp }}$ on $\hat{\omega}$ is less steep and the data more closely resemble theoretical expectations. Among insects, values of $\hat{x}_{0}$ are thought to lie between 0.25 and 0.5 (Ellington, 1984d), although very few studies have attempted to measure this parameter precisely. Within this range of rotational axes, the dependence of $C_{\text {rotexp }}$ on $\hat{\omega}$ is substantial. Nevertheless, as a first approximation, it seems reasonable to model the rotational coefficients as a constant. The validity of this assumption will be tested by the accuracy with which a quasi-steady rotational model based on a constant

\begin{tabular}{|c|c|c|c|c|c|}
\hline $\begin{array}{l}\text { Angular velocity, }(\omega) \\
\left(\operatorname{rad~s}^{-1}\right)\end{array}$ & $\begin{array}{c}\text { Non-dimensional } \\
\text { angular velocity, }(\hat{\omega})\end{array}$ & Slope & $\hat{x}_{0}$-intercept & $C_{\text {rot,exp-intercept }}$ & $r^{2}$ \\
\hline 0.677 & 0.166 & $-1.21 * * *$ & $0.57 * * *$ & 0.69 & 0.80 \\
\hline 0.762 & 0.187 & $-1.35 * *$ & $0.56 * *$ & 0.75 & 0.71 \\
\hline 0.847 & 0.208 & $-1.38 * *$ & $0.65 * *$ & 0.90 & 0.67 \\
\hline 0.931 & 0.229 & $-1.84 * *$ & $0.62 * *$ & 1.14 & 0.87 \\
\hline 1.016 & 0.249 & $-2.24 * *$ & $0.62 * *$ & 1.39 & 0.94 \\
\hline 1.270 & 0.312 & -2.85 & 0.68 & 1.93 & 0.95 \\
\hline 1.354 & 0.333 & -3.13 & 0.68 & 2.13 & 0.97 \\
\hline 1.439 & 0.353 & -3.26 & 0.70 & 2.27 & 0.97 \\
\hline 1.523 & 0.374 & -3.51 & 0.69 & 2.43 & 0.97 \\
\hline
\end{tabular}
force coefficient can predict measured forces.

Table 1. Parameters from $\mathrm{C}_{\text {rot, exp }}$ versus $\hat{\mathrm{x}}_{0}$ regressions for various values of $\omega$

Values for the slope and $x$-intercept that are significantly different from the theoretical predictions based on equation 9 (slope $=-\pi$, $\hat{x}_{0}-$ intercept $=0.75)$ are marked with asterisks $(* P<0.05$, $* * P<0.01, * * * P<0.001)$.

$\hat{x}_{0}$, axis of rotation; $C_{\text {rot, exp }}$, experimental rotational force coefficient. 


\section{Rotational coefficients versus axis of rotation}

As shown in Fig. 2A, the rotational force coefficient $C_{\text {rot,exp }}$ depends on the position of the axis of rotation. Values of $C_{\text {rot,exp }}$ decrease uniformly as the axis of rotation moves from the leading towards the trailing edge, approaching a value of zero for $\hat{x}_{0}=0.75$, as predicted theoretically. Fig. 2B shows $C_{\text {rot,exp }}$ plotted as a function of $\hat{x}_{0}$ at two representative angular velocities $(\hat{\omega}=0.166$ and $\hat{\omega}=0.374)$. For all values of $\omega$, there is a strong linear dependence of $C_{\text {rot,exp }}$ on $\hat{x}_{0}$, with axis of rotation explaining at least $67 \%$ of the variance in $C_{\text {rot,exp }}$ for all cases (Table 1). It is also evident from Fig. 2C (and Table 1) that, as $\hat{\omega}$ increases, the slope, $\mathrm{d} C_{\text {rot,exp }} / \mathrm{d} \hat{x}_{0}$, more closely approaches the theoretical value of $-\pi$ (equation 9 ). The quasisteady model also predicts a value of 0.75 for the $x$-intercept, or critical axis, at which the rotational coefficient changes sign (equation 9). While we could not rotate the wing around this position because of mechanical limitations, the axis that produces zero rotational circulation could be estimated by extrapolation of measured values. As $\hat{\omega}$ increases, the extrapolated estimate for the critical axis closely approaches the theoretical value (Table 1).

\section{Revised quasi-steady model of flapping flight}

We used a single value of the rotational force coefficient $\left(C_{\text {rot,exp }}=1.55\right)$ in the quasi-steady model. This value was based on experiments using the highest measured angular velocity $(\hat{\omega}=0.374)$ and a rotational axis of 0.25 appropriate for our model Drosophila wing. The rotational force estimate was combined in a more comprehensive model that included inertial and translational forces but excluded wake capture. We tested the model against measured forces for 191 diverse kinematic patterns that varied in stroke amplitude, angle of attack, rotational timing and duration (see Sane and Dickinson, 2001). From this assortment, we show three patterns in Fig. 3 that summarize the range of phase relationships between wing translation and rotation and, thus, provide a comprehensive test of the improved quasisteady model.

For each of the three kinematic patterns, the measured lift and drag force traces are compared with the individual inertial, translational and rotational components (Fig. 3). In all cases, the rather small inertial forces account for the sharp undershoots of negative drag conspicuous at the start of stroke reversal. Although the translational quasi-steady model matches the forces during the middle of the stroke
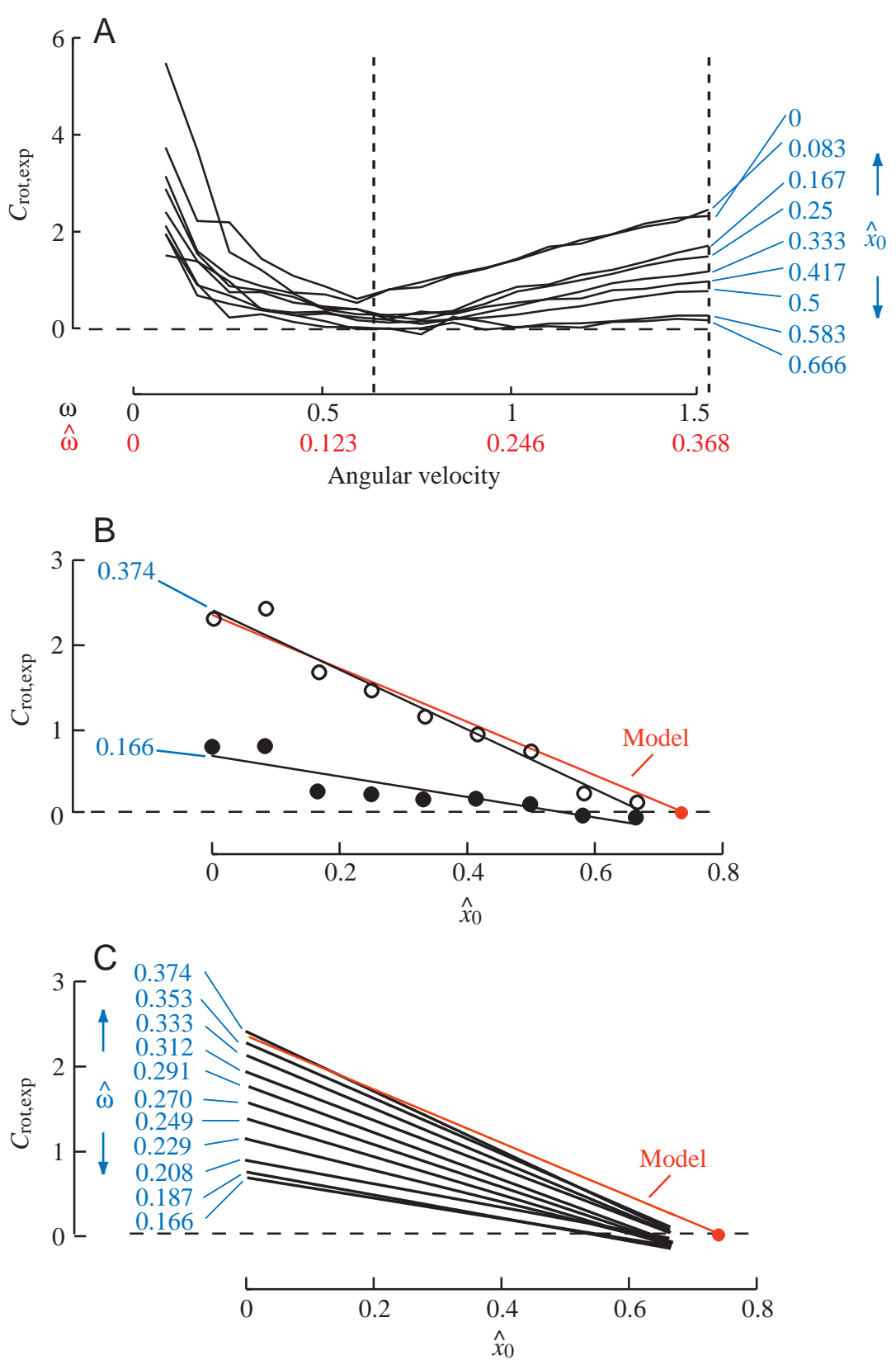

Fig. 2. Variation in rotational coefficient $\left(C_{\text {rot,exp }}\right)$ with angular velocity and axis of rotation. (A) Rotational coefficients versus angular velocity $\left(\omega ; \operatorname{rad~s}^{-1}\right)$ for each axis of rotation. The axes of rotation used for each series of measurements are given in blue. Non-dimensional values of angular velocity $(\hat{\omega})$ are presented in red under the corresponding dimensional value. The vertical dashed lines signify the section through this plot at values of $\hat{\omega}$ of 0.166 and 0.374 , for which the representative regressions are provided in $\mathrm{B}$. $\hat{x}_{0}$, non-dimensional axis of rotation. (B) Rotational coefficient versus axis of rotation. Two representative plots of rotational force coefficients at two values of $\hat{\omega}, 0.166$ (filled circles) and 0.374 (open circles), are plotted, with the corresponding regression lines for each. The red line indicates the prediction given by equation 9. (C) A series of regression lines for representative values of angular velocity (numbers in blue) plotted together with the quasi-steady prediction (red line) from equation 9.

fairly well, it grossly underestimates the forces during wing rotation. The modified quasi-steady model that includes both the rotational and the inertial components shows much better 


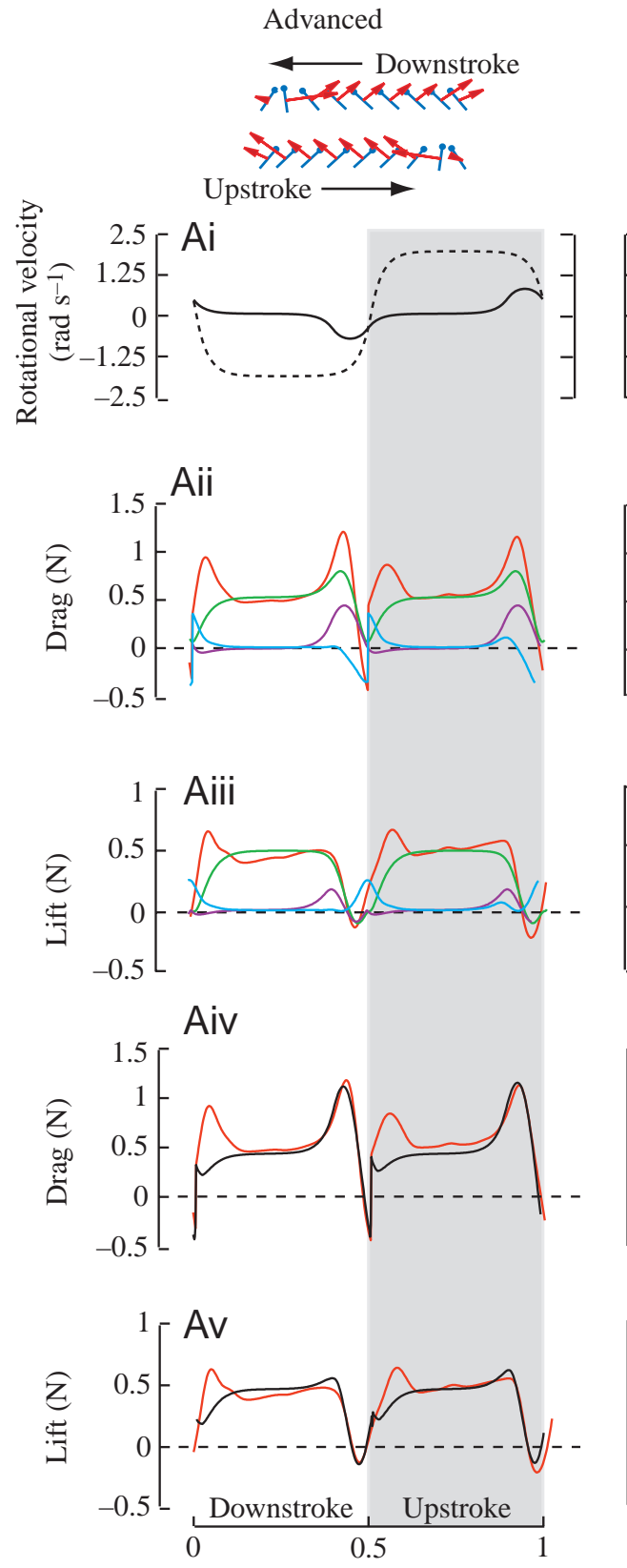

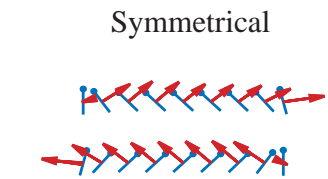


Delayed


Fig. 3. Validation of the revised quasi-steady model for representative kinematic patterns. (A) Advanced rotation. (B) Symmetrical rotation. (C) Delayed rotation. (Ai,Bi,Ci) Two-dimensional cartoons of the three-dimensional kinematics and the translational (broken line) and rotational (solid line) velocities as a function of non-dimensional time within the stroke cycle. The red arrows signify the net force measured on the surface of the flapping wing. (Aii,Bii,Cii) Drag; (Aiii,Biii,Ciii) Lift. The red lines indicate the measured forces, the green lines indicate the quasi-steady translational component based on empirical measurements, the purple lines indicate the quasi-steady rotational component and the blue lines indicate calculated estimates of added mass. In Aiv, v, Biv, v and Civ, v, the summed quasi-steady predictions for drag (Aiv,Biv, Civ) and lift $(\mathrm{Av}, \mathrm{Bv}, \mathrm{Cv})$ are shown in black and compared with the measured values (red lines) for the corresponding kinematics.

agreement with the measured forces for all three representative kinematic patterns. The revised model does not, however, account for the prominent force peaks that follow stroke reversal. These transients, due to wake capture, are fundamentally unsteady and not easily amenable to quasisteady modeling.

\section{Discussion \\ Quasi-steady modeling of insect flight}

Because of the unavailability of instantaneous force data, past researchers have generally focused on the ability of quasisteady assumptions to explain the mean forces required for hovering. To determine the validity of the quasi-steady 
assumption, Ellington (1984a) constructed the following logical argument. If the mean lift coefficient $C_{\mathrm{L}}$ required for hovering exceeds the maximum, measured steady-state $C_{\mathrm{L}}$, then the quasi-steady assumption is contradicted and cannot be used to model insect flight. Alternatively, if the mean $C_{\mathrm{L}}$ does not exceed the maximum, measured $C_{\mathrm{L}}$, then the quasisteady model cannot be discounted. In a comprehensive survey of the aerodynamic data available at the time, Ellington (1984a) observed that, in most cases, the mean values of $C_{\mathrm{L}}$ required to hover were greater than the maximum measured steady-state values of $C_{\mathrm{L}}$. Thus, the quasi-steady model was deemed insufficient to explain insect flight, fostering a search for unsteady aerodynamic phenomena (see also Maxworthy, 1981).

Recent measurements of steady-state forces on model insect wings (Dickinson and Götz, 1993; Dickinson et al., 1999; Sane and Dickinson, 2001) have yielded values for both mean $C_{\mathrm{L}}$ and mean $C_{\mathrm{D}}$ that were substantially higher than values used in previous studies to construct the 'proof-bycontradiction' argument outlined above. In the light of these new data, it is necessary to revisit the quasi-steady approach. Further, although the logic of the 'proof-by-contradiction' approach is rigorous in the realm of time-averaged forces, it is limited in its ability to address the precise time course of the aerodynamic forces (Ellington, 1984a). The data presented here provide evidence that, with the exclusion of forces due to wake capture, a quasi-steady model based on both translation and rotation accurately captures the time history of force generation.

\section{The importance of viscosity in quasi-steady models}

All the force components of equation 2 are based on the assumption of an inviscid fluid. How important is viscosity in models of insect flight at these Reynolds numbers? Recent CFD simulations of Drosophila flight kinematics based on an inviscid model showed strong agreement with simulations based on the full Navier-Stokes equation (R. Ramamurti and W. C. Sandberg, personal communication). Both viscid and inviscid models show reasonable agreement with forces measured on our apparatus using identical kinematics. Further, when we operate the model fly at Reynolds numbers in the range $10^{2}$ and $10^{3}$, the measured forces are similar (data not shown), corroborating the numerical results. Collectively, these results indicate that forces may not be crucially dependent on viscosity within the range of Reynolds number typical for most insects. Other sources of time-dependence, such as the Wagner effect, appear to exert only a minor influence on force generation at these Reynolds numbers (Dickinson and Götz, 1993). Thus, if we take into account the time-variation of flapping kinematics, the resultant translational and rotational force coefficients appear to be time-independent, and it seems reasonable to model them using a quasi-steady approach. It will, however, be necessary to take into account the effects of past strokes on the ambient fluid environment when modeling wing/wake interactions such as wake capture.

\section{Importance of wing rotation in insect flight}

Wing rotation is a necessary feature of flapping kinematics for animals that hover or fly at low advance ratios. Insects actively rotate their wings to manipulate not only the magnitude of aerodynamic forces but also the orientation of the forces with respect to their body. Rapid wing rotations have been observed in most flying insects for which detailed kinematics are available (for a review, see Dudley, 2000). However, precise kinematic measurements are sparse, largely because of the high angular velocities of wing rotation relative to the sampling rates of the methods available to capture kinematics. In Drosophila, in which this behavior has been extensively studied, the timing and duration of wing rotation are actively controlled during visually induced steering maneuvers. In tethered flight simulators, flies advance the rotation of the wing on the outside of a visually elicited turn and delay the rotation of the inside wing (Dickinson et al., 1993). Such advanced and delayed rotations are thought to produce a bilateral force imbalance that should generate a moment to turn the fly in the intended direction.

The range of $\hat{\omega}$ values used in these experiments is approximately an order of magnitude higher than in all previous measurements of which we are aware (see Fung, 1969) and covers a range of values from approximately 0 to 0.4 . Estimates for $\hat{\omega}$ in freely flying Drosophila melanogaster are in the range 0-0.6 (S. Fry, personal communication), and values in other insects may be even higher (see table 2 in Ellington, 1984b). Thus, the experiments conducted here are better suited for modeling insect flight than previous studies based on lower angular velocities of wing rotation.

\section{Comparison between measured and quasi-steady forces}

The quasi-steady model of rotational forces outlined in equations 8 and 9 is based on the assumption of a twodimensional airfoil rotating around an arbitrary axis while translating in an inviscid fluid and is valid only for small magnitudes of angular rotation. Under these simplified conditions, the model predicts that rotational coefficients are independent of the magnitude of the angular velocity. In contrast to these predictions, the measured values of the rotational force coefficients show a clear dependence on angular velocity (Fig. 2). Unlike the quasi-steady model, the measured forces are influenced by the three-dimensional nature of flows, the finite length of the airfoil and the large magnitudes of wing rotation. In the case of two-dimensional flow around a flat plate, sectional aerodynamic force results solely from chordwise circulation, such as that created by an infinitely long vortex filament oriented perpendicular to the wing chord. In the case of a flapping finite wing, vortex filaments must turn along the wing, forming a tip vortex. The presence of a tip vortex may influence the sectional circulation and downwash along the span (Birch and Dickinson, 2001). This distortion of the flow relative to the two-dimensional case may be further complicated by the presence of a base-to-tip axial flow entrained by the leading edge vortex (Ellington et al., 1996). Any or all of these processes may be sensitive to the varying 

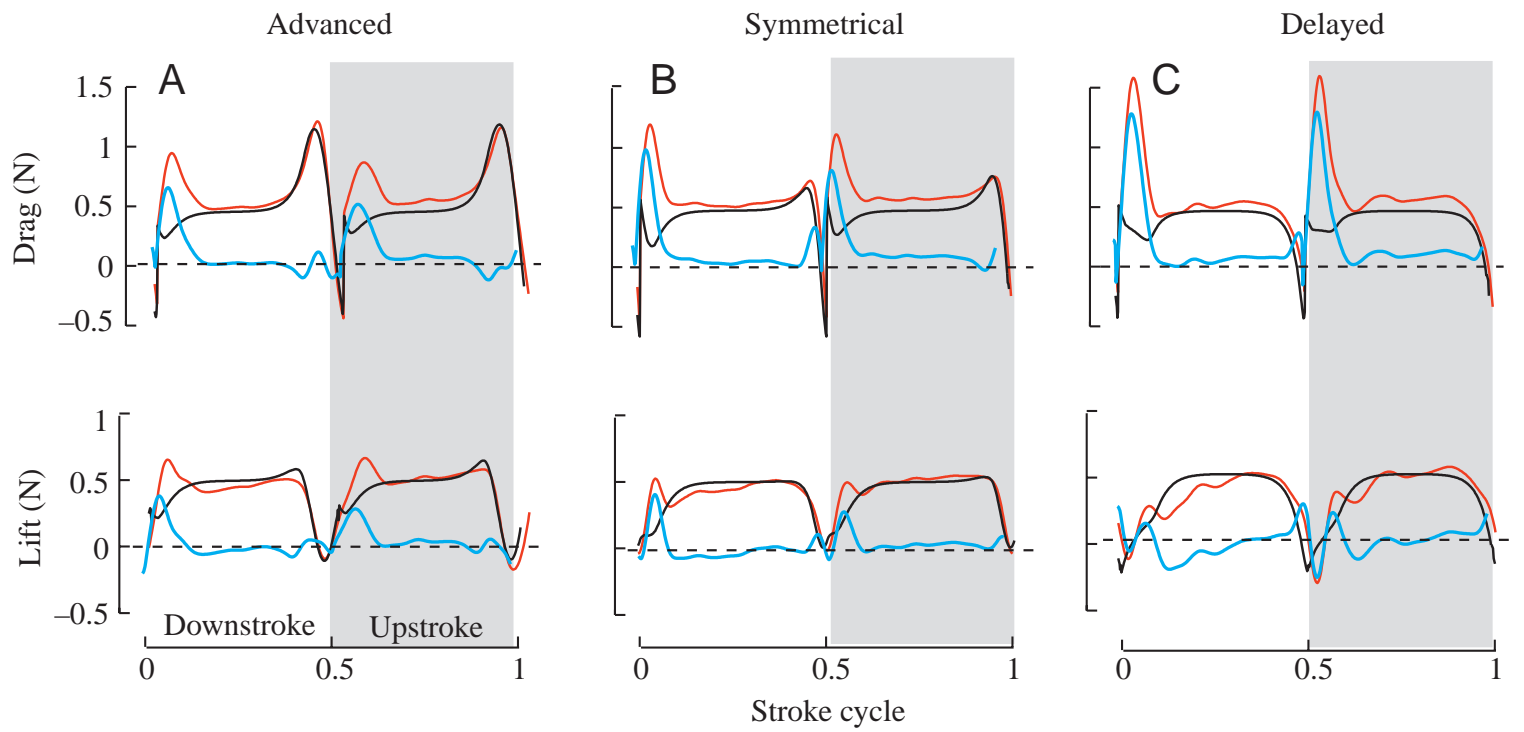

Fig. 4. Isolation of wake capture. (A) Advanced rotation. (B) Symmetrical rotation. (C) Delayed rotation. In all panels, the red line represents the measured forces and the black line represents the sum of the added mass inertia, translational force and rotational force. The force due to wake capture is represented by a blue line and is calculated as the difference between the measured drag (top panels) and lift (bottom panels) and the corresponding quasi-steady estimates.

angular velocity, which could account for the discrepancy between the measured and predicted values. In future experiments, it will be of interest to use flow visualization to observe how either the structure of the tip vortex or the magnitude of the axial flow varies with the magnitude of angular velocity. In spite of this unexpected dependence on angular velocity, a quasi-steady rotational model based on a single, $\omega$-independent value of $C_{\text {rotexp }}$ appears to be sufficient over a broad range of kinematics, as borne out by the close agreement between the modified quasi-steady model and actual force measurements (Fig. 3).

There is better agreement between theory and experiment with respect to the dependence of rotational coefficients on the axis of rotation. As predicted, rotational coefficients show a strong linear dependence on the axis of rotation, even for small angular velocities. At higher angular velocities, for which the experimental data best match the quasi-steady theory, the sign of the rotational coefficient reverses at 0.75 chord lengths, in accordance with theoretical predictions.

\section{Isolation of wake capture}

By rearranging the terms in equation 2, it is possible to use a combination of measured and calculated values to estimate the time course of wake capture forces. An estimate constructed in this manner shows characteristic transients at the beginning of each stroke when the interactions between the wing and the wake are expected to be greatest (Fig. 4). Because the wake capture peak is generated indirectly by subtracting values for the quasi-steady model from actual measurements, this reconstruction is subject to errors arising from inaccuracies within the various quasi-steady components. For example, it is unlikely that the small oscillation in the reconstructed wake capture trace prior to stroke reversal represents an actual interaction between the wing and the wake. More likely, it is an error in the estimate of rotational and inertial forces. These artifacts might diminish if we used values for rotational coefficients that depended on angular velocity or a more accurate model for added mass. Nevertheless, since these errors are typically less than $10 \%$ of the peak force due to wake capture, modeling the rotational coefficients as constants affords us considerable mathematical simplicity at a relatively small cost in accuracy. With a reasonable time course for wake capture, it may be possible to construct a quasi-steady estimate for these more complicated forces as well.

\section{Concluding remarks}

The data presented here characterize the effect of wing rotation on the production of aerodynamic forces by a flapping airfoil. The measured rotational force coefficients show good agreement with the theoretically estimated values and allow us to revise traditional, translation-based models of insect flight by incorporating wing rotation. Compared with traditional models, the revised model shows better agreement with the time course of aerodynamic force generation over a large variety of kinematic patterns. In addition, we can isolate wake capture using the methods outlined here, allowing us to characterize and model the wake capture phenomenon better in future studies.

\section{List of symbols}

c chord length

$\bar{c} \quad$ mean chord length

$\hat{c}(\hat{r}) \quad$ non-dimensional chord length

$C_{\mathrm{D}} \quad$ drag coefficient

$C_{\mathrm{Dt}} \quad$ translational drag coefficient

$C_{\mathrm{L}} \quad$ lift coefficient 


\begin{tabular}{|c|c|}
\hline$C_{\mathrm{Lt}}$ & translational lift coefficient \\
\hline$C_{\text {rot,theo }}$ & theoretical rotational force coefficient \\
\hline$C_{\text {rot,exp }}$ & experimental rotational force coefficient \\
\hline$F_{\mathrm{a}}$ & added mass inertia normal to the wing surface \\
\hline$F_{\text {inst }}$ & instantaneous force normal to the wing surface \\
\hline$F_{\text {rot }}$ & rotational force normal to the wing surface \\
\hline$F_{\text {rot,exp }}$ & $\begin{array}{l}\text { experimental rotational force normal to the wing } \\
\text { surface }\end{array}$ \\
\hline$F_{\text {rot,theo }}$ & $\begin{array}{l}\text { theoretical rotational force normal to the wing } \\
\text { surface }\end{array}$ \\
\hline$F^{\prime}$ rot,theo & $\begin{array}{l}\text { theoretical rotational force per unit span normal to } \\
\text { the wing surface }\end{array}$ \\
\hline$F_{\text {trans }}$ & $\begin{array}{l}\text { instantaneous translational force normal to the } \\
\text { wing surface }\end{array}$ \\
\hline$F_{\mathrm{wc}}$ & wake capture force normal to the wing surface \\
\hline$R$ & wing length \\
\hline$\hat{r}$ & $\begin{array}{l}\text { non-dimensional radial position along the wing } \\
\text { length }\end{array}$ \\
\hline$\hat{r}_{2}^{2}(S)$ & non-dimensional second moment of area \\
\hline$S$ & surface area of wing \\
\hline  & time \\
\hline$U_{\infty}$ & free-stream velocity \\
\hline$U_{\mathrm{t}}$ & wing tip velocity \\
\hline$x_{0}$ & axis of rotation \\
\hline$\hat{x}_{0}$ & non-dimensional axis of rotation \\
\hline$\alpha$ & angle of attack \\
\hline$\Gamma_{\text {rot,theo }}$ & theoretical rotational circulation \\
\hline$\phi$ & angular position of wing \\
\hline$\omega$ & angular velocity of flapping wing $(=\dot{\alpha})$ \\
\hline$\hat{\omega}$ & $\begin{array}{l}\text { non-dimensional angular velocity of flappin } \\
\text { density of fluid }\end{array}$ \\
\hline
\end{tabular}

This work was supported by grants from the NSF (IBN9723424), Defense Advanced Research Projects Agency and the Office of Naval Research (FDN00014-99-1-0892).

\section{References}

Bennett, L. (1970). Insect flight: lift and the rate of change of incidence. Science 167, 177-179.

Birch, J. and Dickinson, M. H. (2001). Spanwise flow and the attachment of the leading-edge vortex. Nature 412, 729-733.

Dickinson, M. H. and Götz, K. G. (1993). Unsteady aerodynamic performance of model wings at low Reynolds numbers. J. Exp. Biol. 174, $45-64$.

Dickinson, M. H., Lehmann, F.-O. and Götz, K. G. (1993). The active control of wing rotation by Drosophila. J. Exp. Biol. 182, 173-189.

Dickinson, M. H., Lehmann, F.-O. and Sane, S. P. (1999). Wing rotation and the aerodynamic basis of insect flight. Science 284, 1954-1960.

Dudley, R. (2000). The Biomechanics of Insect Flight. Princeton, NJ: Princeton University Press.

Ellington, C. P. (1984a). The aerodynamics of hovering insect flight. I. The quasi-steady analysis. Phil. Trans. R. Soc. Lond. B 305, 1-15.

Ellington, C. P. (1984b). The aerodynamics of hovering insect flight. II. Morphological parameters. Phil. Trans. R. Soc. Lond. B 305, 17-40.

Ellington, C. P. (1984c). The aerodynamics of hovering insect flight. III. Kinematics. Phil. Trans. R. Soc. Lond. B 305, 41-78.

Ellington, C. P. (1984d). The aerodynamics of hovering insect flight. IV. Aerodynamic mechanisms. Phil. Trans. R. Soc. Lond. B 305, 79-113.

Ellington, C. P., Vandenberg, C., Willmott, A. and Thomas, A. (1996). Leading-edge vortices in insect flight. Nature 384, 626-630.

Farren, W. S. (1935). The reaction on a wing whose angle of incidence is changing rapidly. Rep. Mem. Aeronaut. Res. Comm. (Great Britain) no. 1648 .

Fung, Y. C. (1969). An Introduction to the Theory of Aeroelasticity. New York: Dover.

Glauert, H. (1929). The force and moment on an oscillating airfoil. Rep. Mem. Aeronaut. Res. Comm. (Great Britain) no. 1561.

Halfman, R. (1951). Experimental aerodynamic derivatives of a sinusoidally oscillating airfoil in two-dimensional flow. NACA TN 2465.

Kramer, M. (1932). Die Zunahme des Maximalauftriebes von Tragflugeln bei plotzlicher Anstellwinkelvergrosserung (Boeneffekt). Z. Flugtech. Motorluftschiff. 23, 185-189.

Liu, H., Ellington, C. P., Kawachi, K., VandenBerg, C. and Willmott, A. P. (1998). A computational fluid dynamic study of hawkmoth hovering. $J$. Exp. Biol. 201, 461-477.

Maxworthy, T. (1981). The fluid dynamics of insect flight. J. Fluid Mech. 93, 47-63.

Munk, M. (1925). Note on the air forces on a wing caused by pitching. NACA TN 217.

Reid, E. (1927). Airfoil lift with changing angle of attack. NACA TN 266.

Sane, S. P. and Dickinson, M. H. (2001). The control of flight force by a flapping wing: lift and drag production. J. Exp. Biol. 204, 2607-2626.

Sedov, L. I. (1965). Two-Dimensional Problems in Hydrodynamics and Aerodynamics, pp. 20-30. New York: Interscience Publishers.

Silverstein, A. and Joyner, U. (1939). Experimental verification of the theory of oscillating airfoils. NACA Report 673.

Theodorsen, T. (1935). General theory of aerodynamic instability and the mechanism of flutter. NACA Report 496.

Walker, P. B. (1931). A new instrument for the measurement of fluid motion; with an application to the development of the flow around the wing started impulsively from rest. Rep. Mem. Aeronaut. Res. (Great Britain) no. 1402 .

Wang, J. (2000). Vortex shedding and frequency selection in flapping flight. J. Fluid Mech. 410, 323-341.

Wilkin, P. J. and Williams, M. H. (1993). Comparison of the instantaneous aerodynamic forces on a sphingid moth with those predicted by quasi-steady aerodynamic theory. Physiol. Zool. 66, 1015-1044. 